\title{
Early planned labor induction vs expectant management in late preterm pre-labor rupture of membranes: maternal and neonatal outcomes
}

\author{
Inshirah Sgayer ${ }^{1,2} \mathbb{D}^{2}$, Karina Naskovica² ${ }^{2}$, Raneen Abu Shaqara2 ${ }^{2}$, \\ Marwan Odeh ${ }^{1,2}$, Jacob Bornstein ${ }^{1,2(0)}$, Maya Frank Wolf ${ }^{1,2}$ \\ ${ }^{1}$ The Azrieli faculty of Medicine, Bar-Ilan University, Safed, Israel \\ ${ }^{2}$ Galilee Medical Center, Nahariya, Israel
}

\begin{abstract}
Objectives: To compare expectant management with early planned labor induction in pregnancies complicated by late preterm pre-labor rupture of membranes (PPROM).

Material and methods: A retrospective file review was conducted in a single tertiary center from January 2015 to September 2019. Singleton pregnancies complicated by late PPROM at 34-36 completed weeks of gestation were enrolled. We compared maternal and neonatal complications between expectant management and early planned labor induction.

Results: We retrospectively assigned 41 women to the expectant management group and 39 to the early planned labor induction group. No difference was found in the mode of delivery between the groups. Women in the expectant management group had a longer antepartum hospital stay compared with the induction group (median of three versus one day, $p<0.01$ ). Neonates were delivered at a more advanced gestational age in the expectant management group compared with that in the induction group ( $355 / 7$ versus $352 / 7$ weeks, $p<0.01$ ). In the induction group, $74.4 \%$ of the neonates were admitted to the intensive care unit (ICU), and $66.7 \%$ received antibiotics compared with $51.2 \%$ of neonates admitted to ICU and $29.3 \%$ receiving antibiotics in the expectant management group $(p=0.04$ and $p<0.01$, respectively).

Conclusions: In pregnancies complicated by late PPROM, early labor induction was associated with a shorter antepartum maternal hospital stay but a higher neonatal ICU admission rate and more frequent antibiotic administration than expectant management. We consider expectant management to be an acceptable alternative to early labor induction in PPROM.

Key words: cesarean section; chorioamnionitis; labor induction; neonatal respiratory distress syndrome; premature rupture of membrane (pregnancy)
\end{abstract}

\section{INTRODUCTION}

Late preterm pre-labor rupture of membranes (PPROM) complicates one percent of pregnancies between the $340 / 6$ and 36 6/7 weeks of gestation [1]. A non-reassuring fetal status, cord prolapse, placental abruption, and chorioamnionitis are all indications for prompt delivery. However, the optimal approach to manage women with late PPROM but without an indication for prompt delivery is still discussed. The American College of Obstetricians and Gynecologists endorse both expectant management and immediate delivery [2]. The Royal College of Obstetricians and Gynecologists considers expectant management to be the best practice management [3].
A recent meta-analysis [4] of 2,563 mothers showed that neonates who were delivered immediately were more frequently diagnosed with respiratory distress syndrome, admitted to the neonatal intensive care unit (NICU), and kept in hospital for prolonged periods of time. In the immediate delivery group, maternal outcomes exhibited a reduced risk of antepartum hemorrhage and chorioamnionitis but an increased rate of cesarean sections (CS).

A multicenter randomized trial demonstrated a significant decrease in neonatal respiratory morbidity in mothers at risk for late preterm delivery who were treated with one course of corticosteroids [5]. Still, to date, there is insufficient evidence regarding the long-term neurodevelopmental

Corresponding author:

Inshirah Sgayer

The Azrieli faculty of Medicine, Bar-Ilan University, 8 Henrietta Szold St, 1311502 Safed, Israel; Galilee Medical Center, Nahariya, Israel

e-mail: inshirah.sg.sh@gmail.com

This article is available in open access under Creative Common Attribution-Non-Commercial-No Derivatives 4.0 International (CC BY-NC-ND 4.0) license, allowing to download articles and share them with others as long as they credit the authors and the publisher, but without permission to change them in any way or use them commercially. 
implications and metabolic risks of such treatment in infants $[6,7]$.

At our institution, we induced labor in all women with late PPROM who then received antibiotic prophylaxis during labor without antenatal corticosteroids administration until 2016. Since 2016, we adopted a different protocol and started offering expectant management that included corticosteroids to women with uncomplicated PPROM until 37 0/7 weeks of gestation.

The aim of this study was to compare the maternal and neonatal outcomes between expectant management and delivery at 34 weeks of gestation in women with PPROM at our institution.

\section{MATERIAL AND METHODS}

The protocol of this retrospective study was approved by the local Institutional Review Board (Helsinki Committee) of the Galilee Medical Center, Nahariya, Israel (date of approval April 28, 2019, number 0041-19-NHR).

We reviewed the records of 186 women with late PPROM at 34-36 completed weeks of gestation who were admitted to our hospital between January 2015 and September 2019. The diagnosis of PPROM was based on the following criteria in either the history or during physical examination: a history of leaking fluid and pooling of amniotic fluid observed on sterile speculum examination. If there was no clear pooling, the AmniSure ROM test (AmniSure International LLC, Boston, MA, US) was used, an immune-chromatography method to detect traces of placental alpha microglobulin-1 protein in the vaginal fluid to confirm the diagnosis.

The exclusion criteria consisted of multiple pregnancy, fetal malformations, meconium-stained amniotic fluid, anhydramnios, non-reassuring fetal status, placental abruption, suspected chorioamnionitis, and active labor or ur- gent CS upon admission. Furthermore, our indications for immediate delivery before the completion of 37 weeks in the expectant management group were a non-reassuring fetal status, cord prolapse, anhydramnios, placental abruption, and chorioamnionitis. In addition, labor was induced if patients refused to continue the conservative follow-up. Based on these criteria, we excluded 58 women with active labor, three with fetal malformations, 57 who underwent immediate cesarean section, and eight in whom labor was induced immediately.

Eventually, 80 cases were enrolled in the study. The patient selection process is described in Figure 1. We divided these patients into two groups: The planned labor induction group consisted of 39 women who underwent planned labor induction with oxytocin (if they did not experience spontaneous labor within 12 hours after PPROM). The expectant management group consisted of 41 women who were monitored and managed expectantly until either spontaneous delivery or 37 0/7 weeks, whatever occurred earlier.

All women were hospitalized from the time of diagnosis until delivery.

The expectant management included:

1. Maternal monitoring for signs of infection including clinical parameters (maternal temperature, presence of uterine tenderness, frequency of contractions, and maternal and fetal heart rate) and laboratory parameters (monitoring white blood cell counts and C-reactive protein every 48 hours).

2. Fetal monitoring included fetal movement count and performing six nonstress tests daily and a biophysical profile twice a week. Oligohydramnios was not considered to be an indication to induce labor.

3. Patients were treated with prophylactic antibiotics for one week, and, from 2017, they additionally received

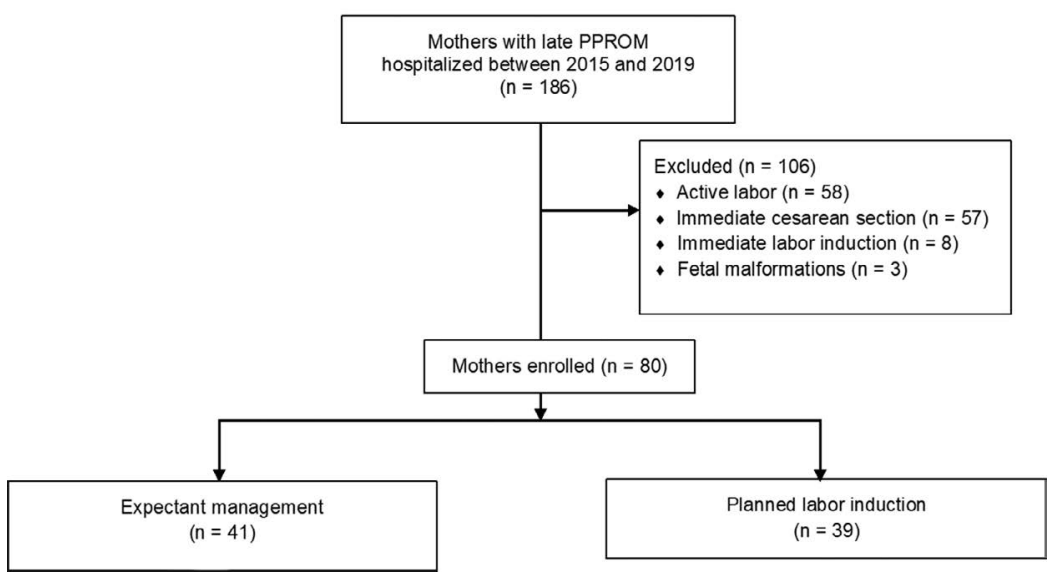

Figure 1. Selection of participants for this comparison of outcomes between early induced labor with expectant management in women with late preterm pre-labor rupture of membranes $(n=80)$; PPROM - preterm pre-labor rupture of membrane 
one course of antenatal corticosteroids at admission if they had not received it previously.

4. Screening for infection included a recto-vaginal swab for a group B strep test and a urine culture that were both obtained on admission.

We recorded information on maternal demographics, the obstetric history, including previous pre-term delivery or PPROM, and the current pregnancy follow-up, including cervical shortening and antenatal corticosteroid administration. Furthermore, we recorded the length of the latency period (time from PPROM until delivery), maternal and fetal monitoring data, whether labor progressed spontaneously or had been induced for medical indications, mode of delivery, rates of antepartum and postpartum hemorrhage (APH and PPH), antepartum fever, intrapartum chorioamnionitis, placental and membrane culture results, antepartum and postpartum hospitalization duration, total hospital stay, postpartum antibiotic administration, and placental histopathological findings. The neonatal outcomes included the Apgar score, birth weight, cord pH, neonatal respiratory support, hospitalization length, data concerning infectious disease evaluation at the NICU, and any complications related to prematurity, such as respiratory distress syndrome, transient tachypnea of newborn, hypoglycemia, intraventricular hemorrhage, necrotizing enterocolitis, hyperbilirubinemia, and stillbirth.

\section{Statistics}

Continuous variables are presented as the mean \pm standard deviation or as median and range. Qualitative variables are presented as frequencies and percentages. Comparisons of continuous variables between the two groups were performed with either the independent sample t-test or Mann-Whitney test based on the sample size of the groups and the variables' distribution shape. Categorical variables were analyzed using Pearson's chi-square or Fisher's exact test. A two-tailed $p$-value $<0.05$ was considered statistically significant.

All statistical analysis was conducted by the statistical department at Galilee Medical Center using IBM SPSS Statistics for Windows, version 25.0 (IBM Corp., Armonk, NY, USA).

\section{RESULTS}

The baseline characteristics of the two groups are represented in Table 1. There were no differences in maternal age, gravity, parity, and the gestational age at admission was similar for the two groups (median of $351 / 7$ weeks). A non-significant trend towards having a history of PPROM or preterm delivery was noted in the induction group compared with the expectant management group (23.1\% versus $7.3 \%$, respectively, $\mathrm{p}=0.06$ ). In the expectant management group, $78 \%$ of women received antenatal steroids compared with only $7.7 \%$ in the labor induction group $(p<0.01)$.

\begin{tabular}{|c|c|c|c|}
\hline & $\begin{array}{l}\text { Expectant management group } \\
\qquad(\mathrm{n}=41)\end{array}$ & $\begin{array}{l}\text { Labor induction group } \\
\qquad(\mathrm{n}=39)\end{array}$ & p value \\
\hline Maternal age, years (mean \pm SD) & $30.20 \pm 6.56$ & $30.59 \pm 5.48$ & 0.77 \\
\hline Gravity (median, range) & $2(1-7)$ & $3(1-8)$ & 0.21 \\
\hline Parity (median, range) & $1(1-5)$ & $2(1-4)$ & 0.19 \\
\hline ART-conceived, n (\%) & $4 / 41(9.8)$ & $1 / 39(2.6)$ & 0.36 \\
\hline History of PPROM or preterm delivery, n (\%) & $3 / 41(7.3)$ & $9 / 39(23.1)$ & 0.06 \\
\hline Cervical shortening, $\mathrm{n}(\%)$ & $2 / 40(4.9)$ & $3 / 39(7.7)$ & 0.67 \\
\hline Previous cesarean delivery, $\mathrm{n}(\%)$ & $4 / 41(9.8)$ & $5 / 39(12.8)$ & 0.73 \\
\hline Thrombophilia, n (\%) & $2 / 41(4.9)$ & $2 / 39(5.1)$ & 1.0 \\
\hline Hypertensive disorders, $\mathrm{n}(\%)$ & $1 / 41(2.4)$ & $0 / 39(0)$ & 1.0 \\
\hline Diabetes, n (\%) & $7 / 41(17.1)$ & $2 / 39(5.1)$ & 0.15 \\
\hline Positive group B streptococcus vaginal swab at admission, $\mathrm{n}(\%)$ & $5 / 23(21.7)$ & $0 / 11(0)$ & 0.15 \\
\hline Gestational age at admission, weeks (mean \pm SD) & $35.13 \pm 0.61$ & $35.10 \pm 0.69$ & 0.87 \\
\hline White blood cell count, median (range) & $11.25(6.01-34.0)$ & $11.08(7.59-15.85)$ & 0.87 \\
\hline C-reactive protein, median (range) & $8(0.90-46.10)$ & $10(0.40-52.0)$ & \\
\hline Steroids given during pregnancy, $\mathrm{n}(\%)$ & $9 / 41(22)$ & $5 / 39(12.8)$ & 0.38 \\
\hline Steroids given at admission, $\mathrm{n}(\%)$ & $32 / 41(78)$ & $3 / 39(7.7)$ & $<0.01$ \\
\hline Duration of PPROM, hours (median, range) & $80.80(25.67-302.60)$ & $20.18(12.38-75.30)$ & $<0.01$ \\
\hline
\end{tabular}

ART — assisted reproductive technology; PPROM — preterm pre-labor rupture of membrane; SD — standard deviation 


\begin{tabular}{|c|c|c|c|}
\hline & $\begin{array}{l}\text { Expectant management group } \\
\qquad(n=41)\end{array}$ & $\begin{array}{l}\text { Labor induction group } \\
\qquad(n=39)\end{array}$ & p value \\
\hline Antepartum LOS in hospital, days, median (range) & $3(1-13)$ & $1(0-3)$ & $<0.01$ \\
\hline Postpartum LOS in hospital, days, median (range) & $3(1-12)$ & $3(2-7)$ & 0.85 \\
\hline Total LOS in hospital, days, median (range) & $7(3-23)$ & $4(3-7)$ & $<0.01$ \\
\hline Antepartum hemorrhage, $n(\%)$ & $1(2.4)$ & $2(5.1)$ & 0.61 \\
\hline Antepartum fever, $\mathrm{n}(\%)$ & $4(9.8)$ & $2(5.1)$ & 0.67 \\
\hline Intrapartum chorioamnionitis, $\mathrm{n}(\%)$ & $/(7.3)$ & $1(2.6)$ & 0.61 \\
\hline \multicolumn{4}{|l|}{ Mode of delivery } \\
\hline Vaginal delivery, $\mathrm{n}(\%)$ & $31(75.6)$ & $32(82.1)$ & \multirow{3}{*}{0.78} \\
\hline Vacuum extraction, $\mathrm{n}(\%)$ & $1(2.4)$ & 0 & \\
\hline Cesarean section, $\mathrm{n}(\%)$ & $9(22.0)$ & $7(17.9)$ & \\
\hline Postpartum hemorrhage, $\mathrm{n}(\%)$ & $0(0)$ & $3(7.7)$ & 0.11 \\
\hline Urinary tract infection, $\mathrm{n}(\%)$ & $1 / 20(4.8)$ & 0 & 1.00 \\
\hline Postpartum antibiotic administration, $\mathrm{n}(\%)$ & $4(9.8)$ & $4(10.3)$ & 1.0 \\
\hline Any positive culture*, $\mathrm{n}(\%)$ & $38 / 40(95.0)$ & $34(87.2)$ & 0.26 \\
\hline Histological chorioamnionitis, $\mathrm{n}(\%)$ & $9 / 24(37.5)$ & $6 / 27(22.2)$ & 0.35 \\
\hline
\end{tabular}

LOS - length of stay; *At least one positive culture of one of the following: uterus, placenta, membranes, or umbilical cord

The median duration of PPROM in the expectant management group was significantly longer compared with the induction group ( 80.80 versus 20.18 hours, respectively, $\mathrm{p}<0.01$ ).

The maternal outcomes are detailed in Table 2 . Women in the expectant management group had a longer antepartum hospital stay than the induction group (median of three versus one day, $p<0.01$ ), with no significant difference in the postpartum hospital stay. Women in the induction group had a shorter total hospital stay than the expectant management group (median of four versus seven days, respectively, $\mathrm{p}<0.01$ ).

In the expectant management group, 24 (58\%) of the women had spontaneous labor prior to 37 weeks, two (4.8\%) underwent induction when they had completed 37 0/7 weeks, and 17 (36\%) underwent labor induction prior to 37 weeks for the following reasons: eight (19.5\%) for suspected fetal distress, four (9.7\%) on maternal request, two (4.8\%) because of severe oligohydramnios, and one (2.4\%) because of suspected chorioamnionitis.

No significant difference was found between the groups in the rates of positive cultures, histological chorioamnionitis, and the different pathogens. Escherichia coli (E. coli) was the most frequently isolated organism in both groups, followed by Enterobacterfaecalis. In addition, Klebsiella pneumoniae, Bacteroides vulgaris, Enterobacter cloacae complex, Bacteroides fragilis and Proteus mirabilis were also isolated.

The neonatal outcomes are shown in Table 3. Neonates were delivered at a more advanced gestational age in the ex- pectant management group compared with the induction group (35 5/7 versus 35 2/7 weeks, respectively, $p<0.01$ ). None of the neonates had a 5-minute Apgar score of less than 7 or $\mathrm{pH}<7.1$ in either group. In the induction group, $74.4 \%$ of the neonates were admitted to the NICU compared with $51.2 \%$ in the expectant management group $(p=0.04)$. In the induction group, $66.7 \%$ of the neonates received antibiotics compared with $29.3 \%$ in the expectant management group $(p<0.01)$.

No case of stillbirth or neonatal death was reported in our study population.

\section{DISCUSSION}

In our population of women with PPROM, planned labor induction was related to higher neonatal ICU admission and antibiotic administration rates than expectant management, while the latter resulted in a longer maternal hospital stay.

These results are in accordance with the findings of previous studies. The PPROMT study [8] was a randomized controlled trial comparing immediate delivery versus expectant management in 1839 women with late PPROM and reported that, compared to labor induction, expectant management resulted in a longer maternal hospital stay since $75 \%$ of these mothers were managed in the hospital until delivery. In our study, all women undergoing expectant management were hospitalized upon admission and, consequently, had longer antepartum and total hospital stays but no difference in the postpartum stay. 
Table 3. Neonatal outcome in 80 pregnancies with late preterm pre-labor rupture of membranes

\begin{tabular}{|c|c|c|c|}
\hline & $\begin{array}{l}\text { Expectant management group } \\
\qquad(n=41)\end{array}$ & $\begin{array}{l}\text { Labor induction group } \\
\qquad(n=39)\end{array}$ & p value \\
\hline Gestational age on delivery, weeks (median \pm SD) & $35.72( \pm 0.76)$ & $35.25( \pm 0.69)$ & $<0.01$ \\
\hline Birth weight, $\mathrm{g}$ (median \pm SD) & $2576.95( \pm 311.21)$ & $2475.18( \pm 263.22)$ & 0.11 \\
\hline Intrauterine growth restriction, $\mathrm{n}(\%)$ & $0(0)$ & $2(5.1)$ & 0.23 \\
\hline Apgar score at $5 \mathrm{~min}$, median (range) & $9(7-10)$ & $9(8-10)$ & 0.84 \\
\hline Umbilical cord pH, median (range) & $\begin{array}{c}7.30 \\
(7.26-7.40)\end{array}$ & $\begin{array}{c}7.31 \\
(7.26-7.37)\end{array}$ & 0.58 \\
\hline Need for resuscitation at birth, $\mathrm{n}(\%)$ & $2(4.9)$ & $1(2.6)$ & 1.00 \\
\hline Admission to NICU, $n$ (\%) & $21(51.2)$ & $29(74.4)$ & 0.04 \\
\hline LOS in NICU, days, median (range) & $8(2-23)$ & $8(2-17)$ & 0.49 \\
\hline Fever at birth & $1(2.4)$ & $2(5.1)$ & 0.61 \\
\hline Antibiotics administration, $\mathrm{n}(\%)$ & $12(29.3)$ & $26(66.7)$ & $<0.01$ \\
\hline Neonatal sepsis, n (\%) & 0 & 0 & - \\
\hline Pneumonia, $\mathrm{n}(\%)$ & 0 & $1(2.6)$ & 0.48 \\
\hline Respiratory distress syndrome, $\mathrm{n}(\%)$ & 0 & 0 & - \\
\hline Transient tachypnea of newborn, $\mathrm{n}(\%)$ & $3(7.3)$ & $1(2.6)$ & 0.61 \\
\hline Invasive ventilation, $\mathrm{n}(\%)$ & $2(4.9)$ & $1(2.6)$ & 1.00 \\
\hline Non-invasive ventilation, $\mathrm{n}(\%)$ & $2(4.9)$ & $2(5.1)$ & 1.00 \\
\hline Surfactant administration, $\mathrm{n}(\%)$ & 0 & $1(2.6)$ & 0.48 \\
\hline Hypoglycemia, n (\%) & $2(4.9)$ & $2(5.1)$ & 1.00 \\
\hline Intraventricular hemorrhage, $\mathrm{n}(\%)$ & 0 & $2(5.1)$ & 0.23 \\
\hline Necrotizing enterocolitis, $\mathrm{n}(\%)$ & 0 & 0 & - \\
\hline Hyperbilirubinemia, n (\%) & $20(48.8)$ & $25(64.1)$ & 0.18 \\
\hline
\end{tabular}

SD — standard deviation; LOS — length of stay; NICU — neonatal intensive care unit

However, in contrast to the PPROMT study [8] and a recent meta-analysis [4] that showed a higher risk of $\mathrm{APH}$ and PPH with expectant management, in our study, there was no difference in the frequency of $\mathrm{APH}$ or $\mathrm{PPH}$ in this group compared to labor induction. These studies included multiple pregnancies, and women who presented with ruptured membranes earlier in pregnancy became eligible upon reaching 34 weeks of gestation and were then followed beyond 34 0/7 weeks. The different inclusion criteria and study design could explain the differences in the results since multiple pregnancies are considered at risk for $\mathrm{PPH}$.

Previous studies reported a higher risk of clinical and histological chorioamnionitis $[9,10]$ in women who underwent expectant management. In contrast, we did not find any difference in maternal infections between the groups. All patients in our study received antepartum antibiotics, either intravenously, orally, or both. In the PPROMT study [8], a similar proportion (86 \%) of patients in both groups received antepartum antibiotics, but women in the expectant management group showed higher rates of intrapartum fever and postpartum antibiotic use. A recent meta-analysis
[11] showed that the use of prophylactic antibiotics is effective in reducing maternal infections in women undergoing expectant management. This discordance between the findings could be related to our small sample size.

PPROMEXIL [9] and PPROMEXIL-2 [10] were randomized controlled trials conducted in the Netherlands and compared labor induction versus expectant management in non-laboring women with PPROM between 34 and 37 weeks. Like our findings, no difference in the CS rate was found between the two approaches. Other studies [4, 8] found higher $\mathrm{CS}$ rates in women with immediate delivery induction. The fact that the Dutch trials involved multiple centers in one country $[9,10]$, while the other trial $[8]$ involved 65 centers in 11 countries. could have affected the CS rate. Both were multicenter studies, but the difference most probably results from different national approaches. For instance, in the PPROMEXIL-2 trial [10], only $13 \%$ of the patients in the induction group underwent CS compared with a twice as high rate of $26 \%$ in the PPROMT study [8]. However, the indications for CS were not reported in these studies, making it difficult to draw definite conclusions concerning the differences in CS rates. 
Similarly, to previous studies $[9,10]$, pregnancy was prolonged by three days in our mothers with expectant management, and their newborns were less likely to be admitted to the NICU. Still, the length of stay of neonates admitted to the NICU was similar in both groups. These findings are in line with previous studies $[4,9]$. Neonatal admission to the NICU results in higher costs and might increase the risk of nosocomial infections [12], but also interrupts the bonding process and increases parental stress [13].

We did not find any difference in neonatal infectious complications (e.g., fever at birth, positive blood cultures, or sepsis) between the groups, but more neonates in the planned induction group received antibiotics than in the expectant management group. Previous studies did not find any difference in neonatal sepsis, positive blood cultures, or antibiotic administration $[4,8,9]$. Furthermore, although in our study neonates in the expectant management group were born at an advanced gestational age, expectant management neither reduced neonatal respiratory morbidity nor affected the need for ventilation. On the opposite, previous studies $[4,8,11]$ showed benefits for expectant management, whereas immediate delivery increased the risk of respiratory distress and mechanical ventilation.

Our data is underpowered to identify differences in neonatal mortality and morbidity between the two approaches, but a recent meta-analysis reported that planned early birth increased the risk of neonatal death (RR 2.55, 95\% Cl 1.17-5.56) without reducing the risk of neonatal sepsis (RR 0.93, $95 \%$ Cl 0.66-1.30) [11].

In our study, $78 \%$ of the women in the expectant management group received one course of steroids at admission compared with only $7.7 \%$ of women in the induction group. This disparity resulted from the new approach introduced in 2017 when we started administering one course of steroids in women with late PPROM [5, 14-16]. Despite this, steroid administration in gestation periods of $\geq 340 / 7$ weeks is still controversial, mainly because of concerns about the long-term neurodevelopmental outcomes in newborns [17].

One of the strengths of this study is that we examined a clearly defined population of women with late PPROM between $340 / 7$ and $366 / 7$ weeks. Previous studies $[8,9,12]$ included women with lower gestational ages at PPROM $(<340 / 7)$ and were followed from 34 weeks onward. Furthermore, detailed maternal and fetal data were recorded over a period of five years. Unlike previous studies, our data were collected from a single center with defined standards of clinical practice, treatment, and care. Despite the relatively small sample size, we could demonstrate a distinct difference in neonatal outcomes, such as higher NICU admission rates and more frequent neonatal antibiotic treatment in the induction group.
Our study has several limitations. As a retrospective cohort study, risk of confounding bias remains even with the use of multivariable statistical techniques. Furthermore, it was underpowered to detect significant differences in certain maternal and fetal complications, such as neonatal sepsis and mortality, because of the small sample size.

\section{CONCLUSIONS}

We found that early planned labor induction in pregnancies complicated by late PPROM was associated with a shorter antepartum maternal hospital stay, but longer neonatal ICU stay and higher rates of antibiotic treatment than expectant management. Thus, we consider expectant management to be a safe and acceptable alternative to early labor induction in PPROM.

\section{Conflict of interest}

None.

\section{REFERENCES}

1. Mercer BM. Preterm premature rupture of the membranes: current approaches to evaluation and management. Obstet Gynecol Clin North Am. 2005; 32(3): 411-428, doi: 10.1016/j.ogc.2005.03.003, indexed in Pubmed: 16125041.

2. Prelabor Rupture of Membranes: ACOG Practice Bulletin Summary, Number 217. Obstet Gynecol. 2020; 135(3): 739-743, doi: 10.1097/AOG.0000000000003701, indexed in Pubmed: 32080044.

3. Thomson AJ. Royal College of Obstetricians and Gynaecologists. Care of Women Presenting with Suspected Preterm Prelabour Rupture of Membranes from 24 Weeks of Gestation: Green-top Guideline No. 73. BJOG. 2019; 126(9): e152-e166, doi: 10.1111/1471-0528.15803, indexed in Pubmed: 31207667.

4. Quist-Nelson J, de Ruigh AA, Seidler AL, et al. Preterm Premature Rupture of Membranes Meta-analysis (PPROMM) Collaboration. Immediate Delivery Compared With Expectant Management in Late Preterm Prelabor Rupture of Membranes: An Individual Participant Data Meta-analysis. Obstet Gynecol. 2018; 131(2): 269-279, doi: 10.1097/AOG.0000000000002447, indexed in Pubmed: 29324621.

5. Gyamfi-Bannerman C, Thom EA, Gyamfi-Bannerman C, et al. NICHD Maternal-Fetal Medicine Units Network. Antenatal Betamethasone for Women at Risk for Late Preterm Delivery. N Engl J Med. 2016; 374(14): 1311-1320, doi: 10.1056/NEJMoa1516783, indexed in Pubmed: 26842679.

6. Committee on Obstetric Practice. Committee Opinion No. 713: Antenatal Corticosteroid Therapy for Fetal Maturation. Obstet Gynecol. 2017; 130(2): e102-e109, doi: 10.1097/AOG.0000000000002237, indexed in Pubmed: 28742678.

7. Kamath-Rayne BD, Rozance PJ, Goldenberg RL, et al. Antenatal corticosteroids beyond 34 weeks gestation: What do we do now? Am J Obstet Gynecol. 2016;215(4):423-430, doi: 10.1016/j.ajog.2016.06.023, indexed in Pubmed: 27342043.

8. Morris JM, Roberts CL, Bowen JR, et al. PPROMT Collaboration. Immediate delivery compared with expectant management after preterm pre-labour rupture of the membranes close to term (PPROMT trial): a randomised controlled trial. Lancet. 2016; 387(10017): 444-452, doi: 10.1016/S0140-6736(15)00724-2, indexed in Pubmed: 26564381.

9. Ham Dv, Vijgen S, Nijhuis J, et al. Induction of Labor versus Expectant Management in Women with Preterm Prelabor Rupture of Membranes between 34 and 37 Weeks: A Randomized Controlled Trial. PLoS Medicine. 2012; 9(4): e1001208, doi: 10.1371/journal. pmed.1001208.

10. van der Ham DP, van der Heyden JL, Opmeer BC, et al. Management of late-preterm premature rupture of membranes: the PPROMEXIL-2 
trial. Am J Obstet Gynecol. 2012; 207(4): 276.e1-276.10, doi: 10.1016/j. ajog.2012.07.024, indexed in Pubmed: 22901981.

11. Bond DM, Middleton $P$, Levett KM, et al. Planned early birth versus expectant management for women with preterm prelabour rupture of membranes prior to 37 weeks' gestation for improving pregnancy outcome. Cochrane Database Syst Rev. 2017; 3: CD004735, doi: 10.1002/14651858.CD004735.pub4, indexed in Pubmed: 28257562.

12. Johnson J, Quach C. Outbreaks in the neonatal ICU: a review of the literature. Curr Opin Infect Dis. 2017; 30(4): 395-403, doi: 10.1097/ QCO.0000000000000383, indexed in Pubmed: 28582313.

13. Chertok IR, McCrone S, Parker D, et al. Review of interventions to reduce stress among mothers of infants in the NICU. Adv Neonatal Care. 2014; 14(1): 30-37, doi: 10.1097/ANC.0000000000000044, indexed in Pubmed: 24472886.

14. Saccone G, Berghella V. Antenatal corticosteroids for maturity of term or near term fetuses: systematic review and meta-analysis of randomized controlled trials. BMJ. 2016; 355: i5044, doi: 10.1136/bmj.i5044, indexed in Pubmed: 27733360.

15. Society for Maternal-Fetal Medicine (SMFM) Publications Committee. Implementation of the use of antenatal corticosteroids in the late preterm birth period in women at risk for preterm delivery. Am J Obstet Gynecol. 2016;215(2): B13-B15, doi: 10.1016/j.ajog.2016.03.013, indexed in Pubmed: 26992737.

16. National Institute for Health and Care Excellence. Preterm labour and birth. Nice guideline [NG25]. Published 20 November 2015. https://www. nice.org.uk/guidance/ng25/chapter/Recommendations\#maternal-corticosteroids (23.01.20218)

17. Stutchfield PR, Whitaker R, Gliddon AE, et al. Behavioural, educational and respiratory outcomes of antenatal betamethasone for term caesarean section (ASTECS trial). Arch Dis Child Fetal Neonatal Ed. 2013; 98(3): F195-F200, doi: 10.1136/archdischild-2012-303157, indexed in Pubmed: 23424017. 